\title{
Long-term trends in foF2: their estimating and origin
}

\author{
J. Laštovička ${ }^{1}$, X. Yue Yu, $^{2,3}$, and W. Wan ${ }^{2}$ \\ ${ }^{1}$ Institute of Atmospheric Physics, Academy of Sciences of the Czech Republic, 14131 Prague, Czech Republic \\ ${ }^{2}$ Institute of Geology and Geophysics, Chinese Academy of Sciences, Beijing 100029, China \\ ${ }^{3}$ Wuhan Institute of Physics and Mathematics, Chinese Academy of Sciences, Wuhan 430071, China \\ ${ }^{4}$ Graduate School, Chinese Academy of Sciences, Beijing 100049, China
}

Received: 17 September 2007 - Revised: 28 January 2008 - Accepted: 6 March 2008 - Published: 26 March 2008

\begin{abstract}
This paper deals with two problems, methods of $f o \mathrm{~F} 2$ trend determination and origin of trends in $f o \mathrm{~F} 2$, both being controversial in current literature. We found that various regression-based methods and artificial neural networkbased method of Yue et al. (2006) provided comparable results within uncertainties caused mainly by various ways of removing/suppressing the dominant solar cycle effect. The role of geomagnetic activity in the observed trends in $f o \mathrm{~F} 2$ was probably substantial and might be still even rather dominant in the last quarter of the 20th century.
\end{abstract}

Keywords. Ionosphere (Ionosphere-atmosphere interactions) - Geomagnetism and paleomagnetism (Time variations, secular and long term) - Radio Science (Ionospheric physics)

\section{Introduction}

The global surface air temperature has been increasing during the recent decades predominantly due to the increasing atmospheric concentration of greenhouse gases. In the upper atmosphere, the radiative effects of greenhouse gases, particularly $\mathrm{CO}_{2}$, become more pronounced and produce a cooling, not a warming effect. The cooling results in the thermal contraction of the upper atmosphere and changes in electron density caused by thermally invoked changes in minor constituents and various reaction rates (Laštovička et al., 2006a). Long-term changes of greenhouse origin may be mixed with that caused by long-term changes of solar and geomagnetic activity, particularly in the ionosphere.

The very important ionospheric parameter is its maximum electron density, represented by $f o \mathrm{~F} 2$, the critical frequency

Correspondence to: J. Laštovička

(jla@ufa.cas.cz) of the F2 layer. Unfortunately, the results of different authors differ substantially, even as to sign and possible origin of long-term change in $f o \mathrm{~F} 2$ (see, e.g., brief review in Laštovička et al., 2006b; Yue et al., 2006). The determination and/or estimation of long-term trends in $f o \mathrm{~F} 2$ are difficult due to the overlapping effect of the 11-year solar cycle, which is many times larger than the effect of long-term trends over the 11 year period. There may be several reasons of differences between trend results as follows: (1) different methods of trend determination, (2) different methods of corrections for solar and geomagnetic activity changes, (3) possible geographic dependence of trends, (4) different periods analyzed, (5) data of different stations and related data quality and homogeneity problems.

In order to estimate the role of the first two factors on trend determination and to explain some differences between results of various authors, six different teams applied their methods (some teams several different methods) of the $f_{o} \mathrm{~F} 2$ trend determination to the same high quality data set (station Juliusruh, northern Germany) over two solar cycles, 19761996 (Laštovička et al., 2006b). Selection of this period starting from solar minimum at the beginning of the first cycle and ending with solar minimum at the end of the second cycle, consists of two very similar consecutive solar cycles (which is very unusual) and, thus, helps to reduce substantially (but not to remove) the effect of solar cycle on trend determination. Various regression methods, wavelet analysis, singular decomposition method, a complex method of Mikhailov et al. (2002), and Danilov's (2002) method of determination of non-geomagnetic component of long-term trend were applied as well as various approaches to corrections for solar and geomagnetic activity effects. Selection of data avoided influence of factors (3), (4) and (5). Results of Laštovička et al. (2006b) may be summarized as follows:

Published by Copernicus Publications on behalf of the European Geosciences Union. 
1. The solar activity correction with proxies F10.7 (observed, not adjusted) and E10.7 is superior to that with sunspot number $R$. Even the optimum selection of the interval under study only partly helps to reduce the effect of the solar cycle on trend determination.

2. The regression-based trends were between about -0.02 and $-0.015 \mathrm{MHz} /$ year; the larger data smoothing (daily, monthly, yearly), the smaller trend. Mikhailov's trends were many times smaller and statistically insignificant. Chilean group wavelet-based (not regressionbased) trends were very small, statistically insignificant, but positive. Two solar cycles with good quality data enable determine trend in yearly values, but such interval is too short to determine reliably seasonal (month-tomonth) variation of trends.

Yue et al. (2006) developed another promising method of trend determination based on artificial neural network and applied it successfully to data from the Asia/Pacific sector. The main purpose of our short paper is to apply Yue et al. (2006) method to the test Juliusruh data set to help to clarify differences between different methods and reliability of different methods and to test if the application of this modern statistical method leads to changes in trend results. Another purpose of the paper is to contribute to the determination of the role of greenhouse gases on long-term trends in $f o \mathrm{~F} 2$.

\section{Trends in $f \circ \mathrm{F} 2$}

High-quality daily values of $f_{o} \mathrm{~F} 2$ observed at station Juliusruh $\left(54.6^{\circ} \mathrm{N}, 13.4^{\circ} \mathrm{E}\right)$ for noon (average from 10:0014:00 UT) are used for the period of two solar cycles from minimum to minimum (1976-1996) to diminish the effect of solar cycle by suitable selection of analyzed data set, as Laštovička et al. (2006b) did. The results obtained by applying the Yue et al. (2006) artificial neural network-based approach with their way of correcting for solar and geomagnetic activity influences to these test data are shown below.

Six combinations of daily values of $R$ (sunspot number), F10.7, E10.7 and geomagnetic activity index $A_{p}$ are used in order to remove and/or suppress the solar cycle and geomagnetic influences on trend determination: (1) F10.7 and $A_{p}$; (2) $R$ and $A_{p}$; (3) E10.7 and $A_{p}$; (4) F10.7 and 11-year running mean $A_{p}$; (5) $R$ and 11-year running mean $A_{p}$; (6) E10.7 and 11-year running mean $A_{p}$. Figure 1 shows daily values of all four indices. It is evident that all three indices describing solar activity do not differ much in longer-term development, while time-development of geomagnetic activity $\left(A_{p}\right)$ differs significantly.

The yearly mean trends for these six combinations of daily values are (in MHz/year):

-0.0103 (F10.7), $-0.0136(R),-0.0162$ (E10.7) for daily $A_{p}$
-0.0155 (F10.7), $0(R),-0.0053$ (E10.7) for 11-year running mean $A_{p}$.

These results illustrate the sensitivity of calculated trends in $f_{o} \mathrm{~F} 2$ to the way how corrections to solar and geomagnetic activity are made. These results also support the result of Laštovička et al. (2006b) that F10.7 and E10.7 are better than $R$ for correcting for solar activity; the former provide more stable results. This is physically plausible because F10.7 and E10.7 describe better the variability of solar EUV radiation than sunspot numbers do. Corrections with the 11-year running mean $A_{p}$ provide worse results (much larger range of results) than when we use daily values of $A_{p}$. As Table 1 and other results of Laštovička et al. (2006b) show, inclusion of correction for $A_{p}$ variability somewhat reduces trend coefficients (by $\sim 10-20 \%$ ) without appreciable impact on variance and standard error, i.e. inclusion of $A_{p}$ mainly slightly increases relative noise in data. Therefore Laštovička et al. (2006b) did not use correction for $A_{p}$; it is simpler without impact on reliability of results. However, their regressionbased trends for F10.7 $+A_{p}$ and $R+A_{p}$ are well comparable with our results here $(-0.0104$ and -0.0147 versus -0.0136 and -0.0103 ), i.e. the neural network method provides results which are not identical but are well comparable with results obtained by simple regression methods. To complete comparison, we computed also trends with only solar correction (without $A_{p}$ correction) (in $\mathrm{MHz} / \mathrm{year}$ ):

-0.0124 (F10.7), $-0.0156(R),-0.0170$ (E10.7).

These trends are really somewhat higher than those including correction to $A_{p}$, as expected, and to some extent question the conclusion about superiority of F10.7 and E10.7 above $R$ for corrections. However, they again essentially agree with trends calculated by Laštovička et al. (2006b).

The above trends without $A_{p}$ correction were computed from daily values. Now we compute trends for monthly mean values instead of daily values of all parameters. Their values (in $\mathrm{MHz} / \mathrm{year}$ ) are as follows:

-0.0094 (F10.7), -0.0074 (R), -0.0162 (E10.7)

These trends are somewhat weaker than trends based on daily values, which is consistent with the fact know to statisticians that smoothing somewhat reduces the magnitude of trends (e.g. E. C. Weatherhead, private communication, 2006), even though the expected difference was smaller. The weakest trends are provided by using sunspot number, $R$, for corrections of solar influence. The trends, however, are still reasonably consistent with trends calculated by other regression-based approaches by other groups in Laštovička et al. (2006b).

Statistical significance of yearly trends is larger than $95 \%$, but this statistically computed significance is only significance with respect to (random) scatter of data. The main problem of results reliability and/or accuracy is due to the correction to solar activity, as illustrated by the above results, and to application of different trend determination methods, as discussed below (if we remove data quality and homogeneity problems). 


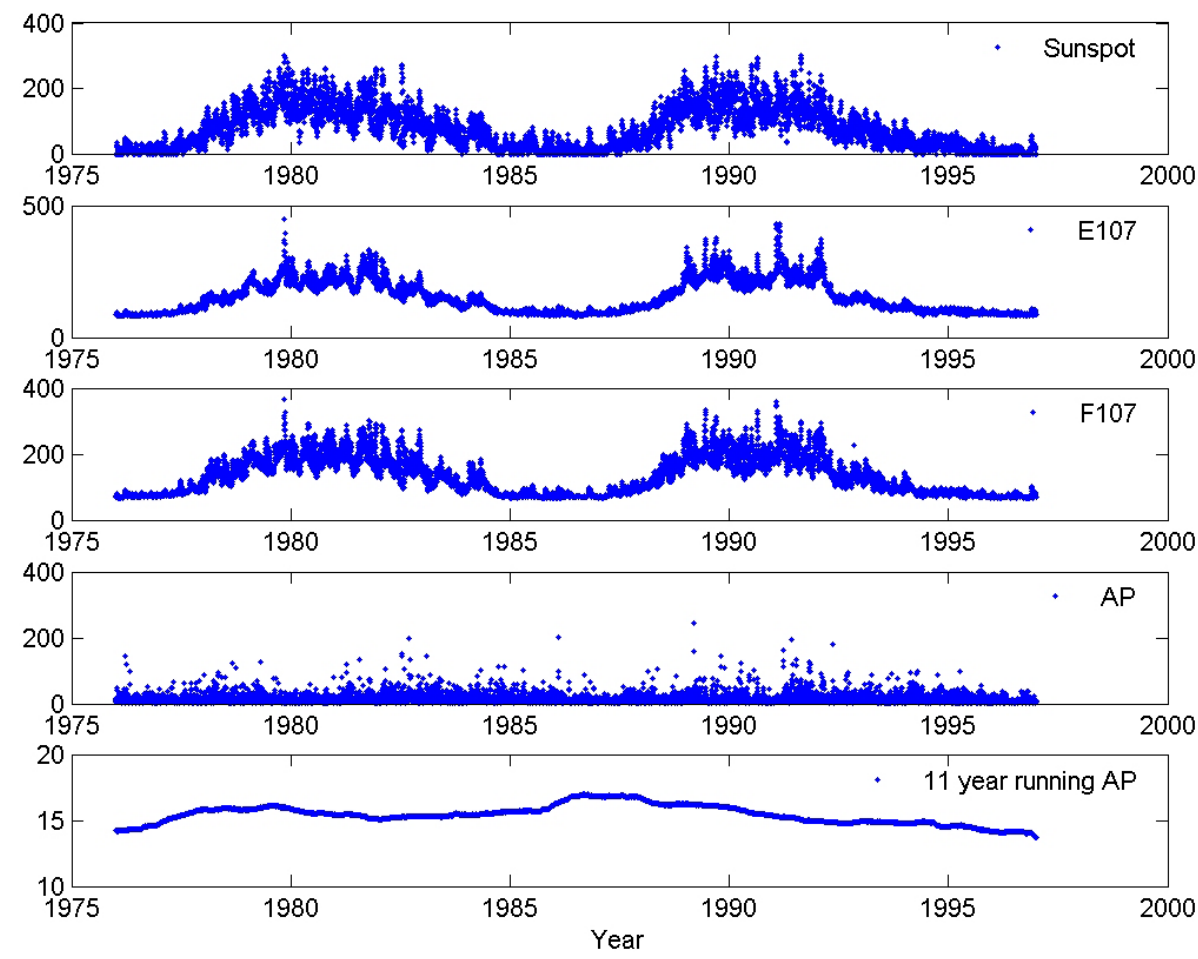

Fig. 1. Course of daily values of solar $(R, \mathrm{~F} 10.7, \mathrm{E} 10.7)$ and geomagnetic activity $\left(A_{p}\right), 1976-1996$.

Laštovička et al. (2006b) found two results, which differed principally from a large group of various essentially consistent regression-based results. Trends determined by a special Mikhailov's method were many times smaller and statistically insignificant as a consequence of his method of correction for the long-term changes in geomagnetic activity. Chilean group's wavelet-based (not regression-based) trends were very small, statistically insignificant, but positive. New careful inspection of Chilean group results reveals very close negative correlation, $r=-0.93$, between their regression-based and wavelet-based monthly trends, and opposite month-to-month variation: positive month-to-month change for regression-based trends is accompanied by negative for wavelet-based trends and vice versa without any exception. This indicates methodological problem in Chilean group wavelet-based trends and their unreliability. Since trends computed by us with the artificial neural networkbased method of Yue et al. (2006) are reasonably consistent with regression-based trends, only Mikhailov's method provides principally different results. Laštovička et al. (2006b) discussed several possible contributors to this difference but each of them was found to play only minor to very minor role.

The final (residual) trends obtained by Mikhailov (2006) by the method introduced by Mikhailov et al. (2002) are very small because the geomagnetic activity effects are practically removed from the observed $f o \mathrm{~F} 2$ variations. If the geomagnetic activity effects were not removed, the Mikhailov's $f o \mathrm{~F} 2$ trends are the same as those obtained by other authors in the paper by Laštovička et al. (2006b) as well as in this paper.

What might be the role of long-term changes of geomagnetic activity in the observed trends in $f o \mathrm{~F} 2$, and what might be the seasonal variation of trends? Figure 2 shows the seasonal variation of trends calculated for monthly mean values of $f o \mathrm{~F} 2, \mathrm{~F} 10.7, R$ and E10.7 without correction to $A_{p}$, with monthly mean $A_{p}$, and with 11-year mean $A_{p}$, respectively. Figure 2 confirms worse performance of correction with $R$, which provides larger scatter of values than corrections with F10.7 and E10.7. It also illustrates predominance of months with relatively weak negative trends, particularly for E10.7 corrections. The impact of geomagnetic activity $\left(A_{p}\right)$ correction on the overall pattern of seasonal variation is small, and as shown above by comparing trend values with and without geomagnetic activity correction, the trends with geomagnetic activity corrections are somewhat lower. However, it might be questioned if our correction to geomagnetic activity is adequate. It should be mentioned that due to the relatively small number of monthly values we consider the seasonal variation to be rather unreliable, at least in absolute values. Laštovička et al. (2006b) obtained very similar seasonal variation as in Fig. 2, but adding of five more years resulted in a substantial change of seasonal pattern of trends towards a more smoothed curve without changing significantly the yearly trend magnitude.

The above results show that the main problem of investigations of long-term trends in $f o \mathrm{~F} 2$ is corrections to solar 


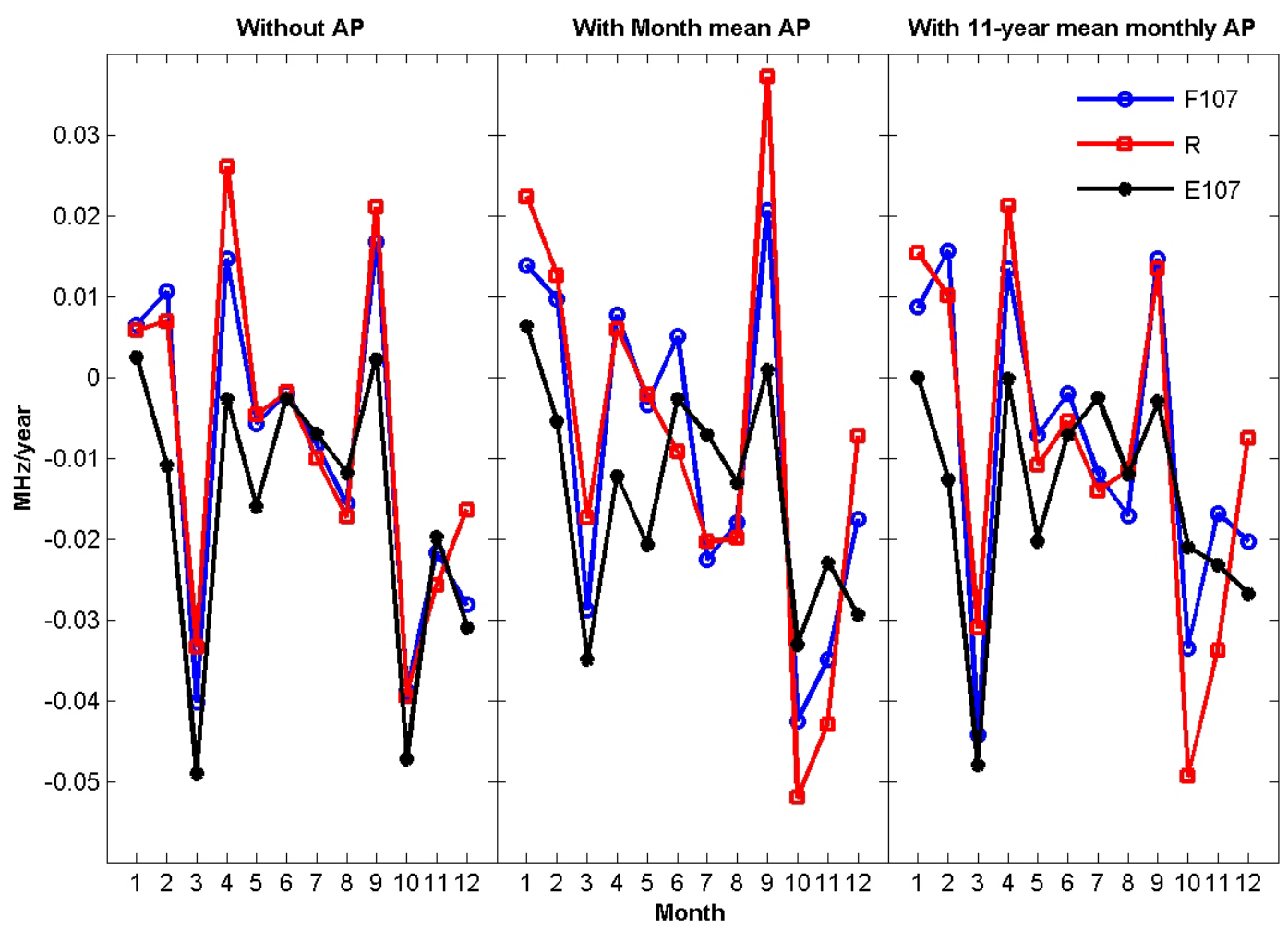

Fig. 2. Seasonal variation of trends based on monthly mean values of $f o \mathrm{~F} 2$ and solar proxies (F10.7 - circles; E10.7 - squares; $R$ - asterisks) for no correction to $A_{p}$ (left panel), correction with monthly mean $A_{p}$ (middle panel), and correction with 11-year mean monthly $A_{p}$ (right panel).

and geomagnetic activity (if we omit data quality problems). The amplitude of solar cycle in $f o \mathrm{~F} 2$ is by $1.5-2$ orders of magnitude larger than the change of $f o \mathrm{~F} 2$ over solar cycle induced by long-term trend for the dataset analyzed in this paper. Therefore, extremely accurate correction for the solar and geomagnetic activities is needed. For solar activity we use various proxies, not the solar ionizing flux, thus some uncertainty always remains, even though for long-term variability the proxies work well (but well does not mean 100\% representation of solar flux). As for geomagnetic activity, various indices of geomagnetic activity, mostly $A_{p}$, have been used. But the geomagnetic activity itself is not driver, it is only indicator/proxy of complex solar wind-magnetosphereionosphere system, i.e. again some uncertainty remains. If the trend and solar cycle would be comparable in magnitude, our corrections would be quite sufficient. However, the trend is much weaker than the solar cycle and, therefore, any very minor inaccuracy in corrections, which is rather probable when we use proxies, can to some degree affect the calculated value of trend. In such situation we should on the one hand search for the best ways of correcting, and on the other hand as climate modelers we should use ensemble approach, i.e. establish a range of acceptable/realistic trend values from various reasonably accurate estimates of trends. Good selection of the interval studied, like here from the solar cycle minimum to the solar cycle minimum of two comparable solar cycles, helps to reduce the problem via "pre-suppression" of the solar cycle influence.

\section{Origin of trends in $f \circ \mathrm{F} 2$}

What is the main driver of trends in $f o \mathrm{~F} 2$ ? The two primary candidates are increasing concentration of greenhouse gases in the atmosphere and increasing geomagnetic activity throughout the 20th century (e.g. Clilverd et al., 2002), and there is a third candidate, specific contamination of the upper atmosphere in the process of space exploration (Danilov, 2006). It is necessary to mention that the mutual role of the greenhouse effect and geomagnetic activity had been changing throughout the 20th century, the relative role of greenhouse effect having been increased in the atmosphereionosphere system from the beginning to the end of the century (Laštovička, 2005).

First model calculations (Rishbeth et al., 1992) revealed some effect of doubled atmospheric $\mathrm{CO}_{2}$ concentration in the height of F2-region peak, $h m \mathrm{~F} 2$, and only a small effect in $f_{o} \mathrm{~F} 2$. The very recent calculations with the top NCAR model TIME-GCM (Qian et al., 2008) provided for greenhouse gasinduced (i.e. non-geomagnetic) trends in the ionosphere positive trends below and negative trends above the transition 
height, which is located slightly below the F2 peak altitude $(h m \mathrm{~F} 2)$. Therefore they obtained weak negative trends in foF2, which were remarkably stronger for solar minimum conditions compared to solar maximum conditions. The proximity of $h m \mathrm{~F} 2$ to the transition altitude and their dependence on solar activity may contribute to the explanation of differences between published results of various authors on trends in $f o \mathrm{~F} 2$.

Danilov's non-geomagnetic trend for the same data set as that used here is about half of full trends computed by others as reported by Laštovička et al. (2006b). Yue et al. (2006) analysis indicates the important role of nongreenhouse factors. Observational results and their physical analysis by Mikhailov (2006) also clearly support the dominance of long-term changes of geomagnetic activity in longterm trends of $f o \mathrm{~F} 2$. Results presented here reveal evidently stronger trends when the correction for geomagnetic activity is not included. This all indicates that the role of geomagnetic activity in the observed trends in $f o \mathrm{~F} 2$ is substantial and may be still dominant even in the last quarter of the 20th century. Therefore $f o \mathrm{~F} 2$ trends can be included with caution or rather not included at present into the global pattern of longterm trends in the upper atmosphere suggested by Laštovička et al. (2006a). In other words, when we search for impact of the increasing concentration of greenhouse gases, we need not care the problems with trends in $f o \mathrm{~F} 2$, as these trends are (or at least were in the past) substantially affected, maybe predominantly governed, by geomagnetic activity. However, the situation can change rather soon in the 21 st century because the atmospheric concentration and therefore the role of greenhouse gases continuously increases and the geomagnetic activity is expected rather terminate its increase.

On the other hand, trends in $f o \mathrm{~F} 2$ are interesting by themselves, because $f_{o} \mathrm{~F} 2$ is one of the principal ionospheric parameters for radio wave propagation, including GNSS systems. Therefore their investigations must continue.

\section{Conclusions}

This paper is focused on two problems, methods of $f o \mathrm{~F} 2$ trend determination/estimation and origin of trends in $f o \mathrm{~F} 2$. Both these topics are controversial in current scientific literature. Our results may be summarized as follows:

1. Methods of determination of trends in $f o \mathrm{~F} 2$. Testing of various methods with a high-quality, reliable data set revealed that various regression-based methods and the neural network-based method of Yue et al. (2006) provide comparable results. Out of two methods, which provided different results, the applied wavelet-based method is unreliable, thus the only method providing quite different results is the Mikhailov et al. (2002) complex method, which among others uses special way of correcting to long-term variability of geomagnetic activity.
2. The solar cycle effect in $f o \mathrm{~F} 2$ is many times larger than the long-term trend in $f o \mathrm{~F} 2$, therefore the results of trend calculations depend on correcting for solar activity, which is made by various ways but always using proxies of solar activity, or geomagnetic activity indices as proxies for solar wind-related activity. For the given test data set the trend revealed by majority of analyses, which includes the effect of long-term change of geomagnetic activity, appears to be between about -0.01 and $-0.02 \mathrm{MHz} / \mathrm{year}$; this "scatter" seems to represent the accuracy with which we are able to determine trends in $f o \mathrm{~F} 2$.

3. Origin of trends. The role of geomagnetic activity in the observed trends in $f o \mathrm{~F} 2$ was probably substantial and might be still even rather dominant in the last quarter of the 20th century. However, the situation can change rather soon in the 21 st century because the atmospheric concentration and therefore the role of greenhouse gases continuously increases and the geomagnetic activity is expected rather terminate its long-term increase.

Acknowledgements. The work of J. Laštovička was supported by the Ministry of Education, Youth and Sports of the Czech Republic through grant OC091 and by the EU project COST296. $X$. Yue was supported by National Science Foundation of China (40636032, 40574071) and National important Basic Research Project (2006CB806306).

Topical Editor M. Pinnock thanks A. Danilov and another anonymous referee for their help in evaluating this paper.

\section{References}

Clilverd, M. A., Clark, T. D. G., Clarke, E., Rishbeth, H., and Ulich, T.: The cause of long-term change in the aa index, J. Geophys. Res., 107(A12), 1441, doi:10.1029/2001JA000501, 2002.

Danilov, A. D.: The method of determination of the long-term trends in the F2-region independent of geomagnetic activity, Ann. Geophys., 20, 511-521, 2002, http://www.ann-geophys.net/20/511/2002/.

Danilov, A. D.: Progress in studies of the trends in the ionosphere F region, Phys. Chem. Earth, 31(1-3), 34-40, 2006.

Laštovička, J.: On the role of solar and geomagnetic activity in long-term trends in the atmosphere-ionosphere system, J. Atmos. Sol.-Terr. Phy., 67, 83-92, 2005.

Laštovička, J., Akmaev, R. A., Beig, G., Bremer, J., and Emmert, J. T.: Global change in the upper atmosphere, Science, 314(5803), 1253-1254, 2006a.

Laštovička, J., Mikhailov, A. V., Ulich, T., Bremer, J., Elias, A. G., Ortiz de Adler, N., Jara, V., Abarca del rio, R., Foppiano, A. J., Ovalle, E., and Danilov, A. D.: Long-term trends in $f o F 2$ : A comparison of various methods, J. Atmos. Sol.-Terr. Phy., 68, 1854-1870, 2006b.

Mikhailov, A. V.: Ionospheric long-term trends: can the geomagnetic control and the greenhouse hypothesis be reconciled?, Ann. Geophys., 24, 2533-2541, 2006, http://www.ann-geophys.net/24/2533/2006/. 
Mikhailov, A. V., Marin, D., Leschinskaya, T. Yu., and Herraiz, M.: A revised approach to $f o \mathrm{~F} 2$ long-term trend analysis, Ann. Geophys., 20, 1663-1675, 2002, http://www.ann-geophys.net/20/1663/2002/.

Rishbeth, H. and Roble, R. G.: Cooling of the upper atmosphere by enhanced greenhouse gases - Modelling of the thermospheric and ionospheric effects, Planet. Space Sci., 40, 1011-1026, 1992.

Qian, L., Solomon, S. C., Roble, R. G., and Kane, T. J.: Model simulations of global change in the ionosphere, Geophys. Res. Lett., doi:10.1029/2007GL033156, in press, 2008.
Yue, X., Wan, W., Liu, L., Ning, B., and Zhao, B.: Applying artificial neural network to derive long-term $f_{o} \mathrm{~F} 2$ trends in the Asia/Pacific sector from ionosonde observations, J. Geophys. Res., 111, A10303, doi:10.1029/2005JA011577, 2006. 